\title{
A CASE OF LEOCODERMA.
}

[Read before the Boston Society for Medical Observation, June 4 th, 1866, and communicated for the Boston Medical and Surgical Journal.]

By Hall Cuntib, M.D., of Boston.

Patient is a short, wiry Irish groom, with a sallow, emaciated, almost cancerous look; he says he has always had rery good health until three weeks ago (he came to me March 4th), when he noticed a small lump in the left groin, which gradually enlarged; not generally painful, though at times there was a sharp sting in it. He also states that he has felt quite chilly at times in the evening, though sitting close to the stove; at night, he is so cold that he licaps coverings on his bed. Awaking, he finds himself sweating profuselysteam rolling up from his arm as he protrudes it from the bedclothes. Three months before this time, he was troubled with occasional dizziness, and would often have fallen if he had not clung to some support.

When I examined him, I found in the left groin an irregular mass lying below Poupart's ligament, stretching obliquely along the line of groin; three inches in length by one and one fourth wide; not uniform, broken as it were into two folds by a furrow running lengthwise with the mass; not tender; hard, elastic, immovable; without fluctuation, impulse, or movement from coughing; not disappearing when on back, or by digital pressure. The skin covering the lower fold of a purplish color, as if pus was superficial. The mass could be nearly surrounded by the fingers.

The mass was certainly irreducible, and it apparently was not crural hernia-though this hernia is not always reducible, its sac being sometimes tied down to the surrounding parts, and changing in its new position to such an extent that it might readily be taken for an inflamed gland.

It was equally improbable that it was a psoas abscess, though the patient now complained of severe pain in his back; however, there were no abnormal features about spine, nor any tenderness at any point; the pain he complained of, situated apparently in the muscles lying on each side of column just below rim of pelvis, preventing his turning when in bed, but not at all troublesome when up, all movements being free. A psoas abscess, formed by pus flowing down from its source higher up, can be emptied by direct pressure; can, so to speak, be reduced.

It was not a bubo. The genitals were free from all suspicious ulcers, cicatrices or discharges. The patient had a gonorrhœa twelve months before. A peculiar discoloration of the penis was noticed, which will be mentioned again.

A varicose state of the femoral can also be reduced, and the hand placed on it can perceive a rustling when the patient coughs. Nothing of the kind was found, though the veins of each leg were in a well-developed varicose state, without ulceration. 
It was judged to be caused neither by a cancerous growth nor by scrofulous glands. The former very rarely attacks the groin primarily. The latter generally are found on each side.

The condition of the man-his loss of flesh, his debility, the sting. ing pain, the rigors and sweating, the gradual development, and the impure state of the blood shown by the unhealthy aspect of abrasions or where splinters had torn the tissues, might cause us to lean' towards the conclusion of its being a cold abscess.

The patient remembered, at last, that he had worn, a short time before he noticed the swelling, a pair of tight boots, which pained him exceedingly, the pain running up to the knee and thigh; so it seems probable that the swelling was caused by the inguinal glands, prone to be excited by the varicose state of the reins, being irritated by the painful constriction of the tight boot, and a subacute inflammation like that of a cold abscess ensuing.

The mass was gradually diminishing, after poultices and strapping. He has never had any inconvenience from it. It has never interfered with his walking. He has been able to work all day till he came to me.

With this, the patient presents the following condition:-skin cool, but breaks readily into perspiration; tongue perfectly clean, moist and.warm; pulse varying from 124 to 84 ; slight cough; no expectoration; pulmonary sounds normal; cardiac at times rapid, continuous, but no valvular abnormal sounds; no headache; no nausea; no vomiting. During treatment, his appetite became excellent; he slept well; had no sweating at night; no recurrence of chills; bowels apt to be constipated.

My attention was attracted to the peculiar state of the skin, and the following notes were taken of a case of Leucoderma.

Patient 40 years of age; his complexion dark brown; hair long, straight, of coal-black lustre, with grey hairs scattered singly, but not occurring in patches; eyes dark brown, with sclerotic coats somewhat congested and yellow; a collar of dark-brown skin encircled the neck, with a concave border, its lower edge broken up into irregular but deeply marked concarities, running just above the clavicles.

The arms, on their posterior aspect, were generally dark (as you would expect to find them in a man who works with his sleeves rolled up); still, here and there, round patches of whitened skin were seen in the midst of the dark integument. The anterior portion of each arm was markedly discolored, this white surface extending irregularly into the dark skin of posterior aspect. Notling peculiar about hands, cxcepting a few unhealthy ulcers from splinters. The chest and back were of a dirty mottle, the latter retaining its color better than the chest. The whitened skin was most marked over the abdominal region, where it spread largely, breaking into the natural dark pigment-skin of the lateral portion of the body. The integument about the umbilicus preserved its natural color, though 
looking of deeper hue than the surrounding whitened skin, and being more pronounced on the lateral and lower parts, yet broken up, and fading into white.

The white patches encroached upon the dark skin, which lay along the right side, stretching almost in a continuous surface from the right crest of ilium up to axilla, dotted here and there with islands of white of varying shape and size. The axillæ were white. On the left side, the whitened patches had spread much more; only a dark patch remained over left iliac crest, and a narrow stripe in left iliac region.

The whitened patches ended in an irregular convex border on each thigh at the upper fifth. The legs were dark brown, with the exception of the popliteal spaces where the white tints again appeared. The upper two-thirds of penis were white, the lower third of prepuce dark brown-the upper portion of scrotum white and covered with white hairs. The pubis mostly covered with dark hair. On the lower part of scrotum where the skin was dark, the white hair seemed to be nearly equal to the dark.

On the centre of the lower lip was a dark colored patch, the size of half a pea.

It seems to me this case would be classed by many with the bronzed skin or Addison's disease-many of its prominent features resemble it, and might lead to that conclusion. Thus Dr. Addison writes"The leading characteristic features of the morbid state to which I wish to call attention are anæmia, general languor and debility, remarkable feebleness of the heart's action, irritation of the stomach, and a peculiar change of color in the skin occurring in connection with a diseased condition of the supra-renal capsules. Now in this case the irritation of stomach is the only point which differs. The patient has a good appetite, good digestion, no nausea, no vomiting."

Dr. Addison again says, the discoloration pervades the whole surface of body, but is commonly most strongly manifested on the face, neck, superior extremities, penis and scrotum, and in the flexures of the axillæ and around the naval. In this case the deep coloration is found strongly marked, if you will, on the neck, superior extremities, naval, penis and scrotum, but the flexures of the axillæ and the popliteæ are light.

The discoloration has a method of affecting the body by a uniform discoloration of the whole integument; of course most marked where there is the most pigment- thus the axillæ and pubis are prominently affected. The face and hands also being exposed to the sun, eárly show the change. "In some cases the discoloration occurs in patches, or perhaps certain parts are so much darker than others, as to impart to the surface a mottled or somewhat chequered appearance." Dr. Wilks, of Guy's Hospital Reports of 1862, does not deny that the color may occur in patches, but he thinks he is correct in saying that all experience has hitherto shown that the discolora- 
tion has been uniform over the whole of the body. In fact, in many of the cases given to disprove Addison's disease, the pigmentation has occurred in patches, and in others there can be no doubt that jaundice, pityriasis, ephelis, and icthyosis have been mistaken for the diseoloration. Of the 25 cases given by Dr. Wilks, all were very dark except four, where discoloration was slight, and one where there was no discoloration.

Pain in back is found in sercral cases. In three cases the spine was diseased and psoas abscess formed. In most cases the pain was situated in the gastric region, passing from the epigastrium through to the shoulders.

In the monagraph of Dr. Addison it is stated, that symptoms may be observed in the course of the disease which denote disturbed cerebral circulation. Dr. Wilks more strictly considers the nervous centres to be impaired, and that the various symptoms result therefrom. Thus in these cases reported by him-delirium and convulsions occurred in 2-numbness of fingers in 2-one of them accompanied by neuralgic pains in limbs and delirium. In this case there were sudden attacks of dizziness with tendency to fall. In one case reported in the Medical Times, Jan. 24, 1857, by Dr. Barlow, loss of consciousness, and what are termed "fainting fits," were the earliest symptoms noticed.

Dr. Hutchinson, in London Hospital Reports for 1864, sums up the matter in the following words: "In morbus Addisonii we have a diffused darkening of the skin, with spanæmia, and greatly enfeebled health. The skin becomes darkest on the parts naturally supplied most liberally with pigment, on those exposed to the sun and air, or to accidental irritants (blisters, \&c.); a disagreeable negro-like odor often attends the patient. No where except on mucous surfaces are there any abruptly margined patches."

In pityriasis versicolor, we lave dark brown patches with well defined spreading borders on normally tinted skin. The disease is rare in the aged, and never occurs in children. The patches rarely occur in other places than the chest, back, abdomen, shoulders and upper extremities. A cryptogam can be readily demonstrated by the microscope.

In leucoderma, we have perfectly (white, well defined patches, with spreading edges on a brown skin. The disease may affect any part, and is common on the forearms and hands. It may occur at any age. There is no cryptogam.

Dr. Watson has apparently confounded the two diseases, for in his description of " bronzed skin," he says, "sometimes the discoloration is deeper here and there, and gives a mottled appearance to the surface, and sometimes it is diversified with white patches in which the skin is blanched, is more white and colorless than healthy skin, like the nails and conjunctive, and the hair on the head, and on the pubes, corresponding to those spots, becomes perfectly white. 
As Dr."Hutchinson writes, "Lcucoderma commences in white points - these enlarge into small round patches-these increase, coalesce, and thus form irregularly shaped patches, but yet pursuing the convex border. Sometimes small islets of brown may be left on large areas of white." In our case at first sight the dark patches seemed much more apparent than the white, as if they constituted the abnormal state, but on closer examination they (the dark portions) constantly showed the concave border, and very markedly so; they were smooth, they were not raised above the skin, there were no loose scales of epidermis, nor any cryptogams, nor was there any itching. The white patches, whether as dots, as small separate patches, or largely spreading tracts, always presented the convex border, breaking into the natural dark skin, which splitting up before them seemed to melt away at their approach.

The patient said he had noticed these discolored spots first when a boy, some 30 years ago, when bathing in Ireland with his brother. Their attention was attracted by the white appearance of the thighs and side of chest.

\section{Zheports of fterical Societies.}

EXTRACTS FROM THE RECORDS OF THE BOSTON SOCIETY FOR MEDICAL IAPROVEMENT. BY CHARLES D. HOMANS, M.D., SECRETARY.

Apsil 23d.-Cancer of the upper part of the Essophagus, involving the Larynx, and opening exlernally.-Dr. JACKson showed the specimen, which Dr. Henry Cowles, of Saxonville, had brought to him this morning, and which was removed yesterday. The patient was a tall, thin, dark-complexioned, healthy woman, and knew of no case of cancer in her family. Age, 70 years. Rather more than a year ago, she began to have soreness of the throat, with dysphagia, and this went on increasing until death, so that from the time when Dr. C. first saw her, nine weeks ago, she could take no solid food. There was then a firm swelling, in the form of a ring, over the upper part of the larynx, and along the left side of the neck, extending somewhat to the right side, discolored, and looking like an abscess that would soon break. In a few days it opened like a carbuncle, by three or four small apertures, and discharged a considerable quantity of thin pus. About two weeks afterwards, a probe was passed in towards the left side, and over two inches; and $\mathrm{Dr}$. C., suspecting a communication with the diseased surface, gave the patient a little indigo-water, which soon appeared externally and verified his diagnosis. Towards the last, about one third of all the liquid she took was discharged externally, but none seemed to pass into the trachea, so as to cause choking, un. til the last few days. The pain and soreness was very great, extending into the left side of the neck and ear. Much mucus hawked from the throat, and during the last two days some blood. From early in her sickness she was hoarse, and occasionally quite so, but never aphonic. No dyspnœa until the last three weeks, but afterwards in paroxysms 